June M. de la Cruz $\cdot$ Richard N. Bamford

Rebecca D. Burdine $\cdot$ Erich Roessler

A. James Barkovich · Dian Donnai · Alexander F. Schier

Maximilian Muenke

\title{
A loss-of-function mutation in the CFC domain of TDGF1 is associated with human forebrain defects
}

Received: 12 December 2001 / Accepted: 6 February 2002 / Published online: 10 April 2002

(C) Springer-Verlag 2002

\begin{abstract}
TDGF1 (CRIPTO) is an EGF-CFC family member and an obligate co-receptor involved in NODAL signaling, a developmental program implicated in midline, forebrain, and left-right axis development in model organisms. Previous studies of $C F C 1$ (CRYPTIC), another member of the EGF-CFC family, demonstrated that normal function of this protein is required for proper laterality development in humans. Here we identify a mutation in the conserved CFC domain of TDGF1 in a patient with midline anomalies of the forebrain. The mutant protein is inactive in a zebrafish rescue assay, indicating a role for TDGF1 in human midline and forebrain development.
\end{abstract}

\section{Introduction}

Holoprosencephaly (HPE) is the most common congenital malformation of the forebrain and is associated with various etiologies, including environmental and genetic factors. The pathogenesis of HPE, or the related phenotype in animal models, cyclopia, can be traced to the defective development or function of the axial midline during gastrulation, which is required to split the single eye field and

J.M. de la Cruz $\cdot$ R.N. Bamford · E. Roessler $\cdot$ M. Muenke (®) Medical Genetics Branch,

National Human Genome Research Institute,

National Institutes of Health, 10 Center Drive, MSC 1852,

Building 10, 10C103, Bethesda, MD 20892-1852, USA

e-mail: muenke@nih.gov,

Tel.: +1-301-4028167, Fax: +1-301-4807876

R.D. Burdine · A.F. Schier

Developmental Genetics Program,

Skirball Institute of Biomolecular Medicine,

Department of Cell Biology,

New York University School of Medicine, New York, USA

A.J. Barkovich

Department of Neuroradiology,

University of California San Francisco, California, USA

D. Donnai

Regional Genetic Service, St. Mary's Hospital, Manchester, UK brain into left and right halves. The spectrum of HPE, as seen in humans, is tremendous and ranges from the most extreme cases with a single eye associated with an undivided cerebral cortex to mild microforms consisting of subtle midline features, such as a single central incisor (Muenke and Beachy 2001). Our understanding of the pathogenesis of cyclopia in animal models has led to experimental verification that many of the genes implicated in these model systems also are predictive of human HPE genes necessary for midline development (Roessler and Muenke 2001). For example, defects in the Sonic Hedgehog pathway are associated with cyclopia in all vertebrates examined, and are also the most common cause to date of familial HPE in humans (Nanni et al. 1999). The Hedgehog class of proteins constitutes secreted factors with patterning activity in the early embryo, and such signaling from the anterior midline prechordal plate cells is thought to directly act on the presumptive telencephalon and eye field to induce their division. In addition to factors that are directly associated with this division, there are genes such as Nodal that are required much earlier during development, yet genetic studies reveal that defective function can also result in cyclopic phenotypes. This can be best understood by the requirement for Nodal function in the specification of the endomesoderm, whose derivatives, including the axial midline cells that will produce Sonic Hedgehog, participate in the division of the eye field and brain (Shen and Schier 2000).

During early embryonic development, Nodal is a key signaling factor involved in the organization and specification of the vertebrate body plan (Schier and Shen 2000; Gritsman et al. 2000; Burdine and Schier 2000). Nodal signals are implicated in mesoderm formation, specification of the anterior-posterior axis, the left-right axis, and neural patterning and are considered to act by binding and activating the Activin-like serine/threonine receptor complex (Zhou et al. 1993; Conlon et al. 1994; Shen et al. 1997; Zhang et al. 1998; Feldman et al. 1998; Schier et al. 1997; Schier and Talbot 2001). In this pathway, the EGFCFC family of proteins acts as obligate cofactors for Nodal signaling. Members of this family, which include the ze- 
brafish one-eyed pinhead (oep), murine Tdgf1 and Cfc1, as well as frog Frl-1, are membrane-associated, glycosylated proteins that are characteristically defined by a variant EGF-like motif and a novel cysteine-rich region or CFC domain (Shen et al. 1997; Zhang et al. 1998; Shen and Schier 2000). In the case of Tdgf1 (Cripto), recent biochemical studies in the mouse have shown that the binding of Nodal to its receptor complex is, in fact, facilitated by the CFC and EGF-like domains of the Tdgf1 molecule (Yeo and Whitman 2001). These studies demonstrated that the CFC domain allows Tdgf1 to bind as a coreceptor to the Activin-like type I serine/threonine kinase receptor, while the EGF domain permits Nodal's association to an Activin-like type II receptor. Consequently, the activated serine/threonine kinase of the Nodal-bound receptor complex targets critical downstream substrates, including the transcription factor Smad2 (Yeo and Whitman 2001).

Studies in zebrafish show that oep-deficient embryos develop severe germ-layer defects, have a malformed anterior-posterior (A-P) axis, and are severely cyclopic and heterotaxic, emphasizing that oep is required for both midline and laterality development (Schier et al. 1997; Strahle et al. 1997; Zhang et al. 1998; Gritsman et al. 1999; Yan et al. 1999; Gritsman et al. 2000; Schier and Shen 2000). Furthermore, other members of the EGF-CFC family, including mouse $T d g f 1$, human and mouse $C F C 1 / C f c 1$, and frog Frl-1 can rescue the oep phenotype, indicating similar biological roles for all members of the EGF-CFC family (Gritsman et al. 1999; Bamford et al. 2000).

In contrast to the single EGF-CFC gene in zebrafish (oep), higher vertebrates have two genes, namely $T d g f 1$ and $C f c l$, each with distinguishable phenotypes in knockout studies. $C f c l$ mutant mice have left-right axis defects, including right isomerism and heterotaxia (Shen et al. 1997; Yan et al. 1999), whereas mice null for $T d g f l$ have a mispositioned A-P axis and lack embryonic mesoderm and endoderm (Ding et al. 1998). In addition, Tdgfl mutant mice do not form a primitive streak, a major prerequisite structure for proper prechordal plate development and, subsequently, to the division of the forebrain (Ding et al.
1998; Shen and Schier 2000; Burdine and Schier 2000; Roessler and Muenke 2001). However, since Tdgfl mutant mice are embryonic lethals, a direct correlation between the genotype of these mice and the development of cyclopia, other forebrain anomalies, or laterality cannot be demonstrated.

Mouse studies show that $C f c l$ expression is primarily localized to the anterior primitive streak and lateral plate mesoderm (Shen et al. 1997; Shen and Schier 2000). We had previously studied human $C F C 1$ for its potential role in holoprosencephaly (HPE). However, as suggested by the subsequent mouse studies, no $C F C l$ mutations were detected in human HPE patients, but were readily identified in patients with left-right axis defects. Among the loss-offunction mutations found were R112C, a missense mutation of a highly conserved residue in the EGF-like motif, and G174del1, which alters the reading frame of the carboxy terminus of the protein (Bamford et al. 2000). Our studies suggested that the primary role of human $C F C 1$ is in laterality development and that there might be a potential role for human TDGF1 in normal axial midline development. By default, TDGF1, became a promising candidate gene for midline disorders such as HPE.

\section{Materials and methods}

\section{Patient samples}

Genomic DNA was extracted by standard methods from either blood or lymphoblastoid cell lines from a panel of 83 familial and 327 sporadic cases of HPE. This patient collection represents all of the HPE cases presently available to the lab and is known to include cases representing all degrees of clinical severity. All samples were obtained according to the guidelines of the Institutional Review Board of the National Human Genome Research Institute, NIH.

\section{Polymerase chain reaction analysis}

Amplification of genomic DNA was performed in a $30-\mu 1$ reaction volume, using 60-100 ng DNA template, $50 \mu \mathrm{M}$ dNTP, $0.25 \mu \mathrm{M}$ of each primer, $3 \mu \mathrm{l}$ of $10 \times \mathrm{PCR}$ amplification buffer (Gibco, Md.), $1.5 \mu \mathrm{l} 10 \times e n h a n c e r$ buffer (Gibco), $0.9 \mu \mathrm{l}$ of $50 \mathrm{mM}$ of $\mathrm{MgSO}_{4}$,
Table 1 Amplification primers, conditions and dHPLC parameters

\begin{tabular}{llll}
\hline Exons $\left(5^{\prime}-3^{\prime}\right)$ & Size $(\mathrm{bp})$ & $\begin{array}{l}\text { PCR annealing } \\
\text { temp }\left({ }^{\circ} \mathrm{C}\right)\end{array}$ & $\begin{array}{l}\text { dHPLC } \\
\text { temp }\left({ }^{\circ} \mathrm{C}\right)\end{array}$ \\
\hline $\begin{array}{l}\text { Exon 1: } \\
\text { GGGTTTGTGTTGAAGAAGG }\end{array}$ & 359 & 54 & 58 \\
$\begin{array}{l}\text { CCTAAGCTGGCAATCTCTAGC } \\
\text { Exon 2/3: }\end{array}$ & 335 & 52 & 61 \\
$\begin{array}{l}\text { TTTGGCTAACTCATGTTTGACTTCC } \\
\text { GCAGAAGCATCGAAGTCAGGC }\end{array}$ & & & \\
$\begin{array}{l}\text { Exon 4/5: } \\
\text { GCACTTTCCATCCCTACACC }\end{array}$ & 401 & 54 & 59,61 \\
$\begin{array}{l}\text { GGCAAAAGAAAGAGGAGAACC } \\
\text { Exon 6: } \\
\text { GACCAAGCATCCCTACCTTC } \\
\text { TGTAGCAGCAGCCTTTACTG }\end{array}$ & 218 & & \\
\hline
\end{tabular}


and $1 \mathrm{U}$ AmpliTaq (Perkin Elmer, Calif.). All the reactions were performed in PTC-225 thermocycler (MJ Research, Mass.). Primer pairs and respective annealing temperature are shown in Table 1. PCR cycling parameters were $95^{\circ} \mathrm{C}$ for $4 \mathrm{~min}$, followed by $30 \mathrm{cy}-$ cles at $95^{\circ} \mathrm{C}$ for $30 \mathrm{~s}$, annealing at the indicated temperatures, respectively, for $30 \mathrm{~s}$ and $72{ }^{\circ} \mathrm{C}$ for $1 \mathrm{~min}$, with a final step of $72{ }^{\circ} \mathrm{C}$ for $5 \mathrm{~min}$. One half of the PCR products was used for dHPLC analysis and the other half was stored for direct DNA sequencing.

Denaturing high-pressure liquid chromatography screening

Denaturing high-pressure liquid chromatography (dHPLC) was performed on a WAVE (Transgenomic, Calif.) instrument. In order to enhance heteroduplex formation, the PCR products were denatured at $95^{\circ} \mathrm{C}$ for $5 \mathrm{~min}$, followed by gradual cooling to $60^{\circ} \mathrm{C}$ over $15 \mathrm{~min}\left(1^{\circ} \mathrm{C} / 30 \mathrm{~s}\right)$. PCR products were automatically injected onto a DNAsep column and eluted with a linear gradient of buffers $\mathrm{A}$ and $\mathrm{B}[\mathrm{A}=100 \mathrm{mM}$ triethylamine acetate $(\mathrm{pH} 7) ; \mathrm{B}=100 \mathrm{mM}$ triethylamine acetate $(\mathrm{pH} 7), 25 \%(\mathrm{v} / \mathrm{v})$ acetonitrile] at a constant rate of $0.9 \mathrm{ml} / \mathrm{min}$. Samples were analyzed at the melting temperature $(\mathrm{Tm})$ determined by using the WAVEMAKER software (Transgenomic, Calif.). Heterozygous profiles were identified by visual inspection of the chromatograms on the basis of the appearance of additional earlier eluting peaks. Corresponding profiles for the same amplicons that exhibited only a single peak could be demonstrated by additional studies to be homozygous and normal in sequence. The GC contents of amplicons spanning exons 4, 5 , and 6 was high at one particular region; hence, the dHPLC analysis was repeated at a raised temperature to make the analysis more sensitive in detecting the heteroduplexes.

\section{Direct DNA sequencing}

Amplicons displaying heterozygous profiles were purified using a Qiagen PCR purification kit (Qiagen, Calif.) and bi-directionally sequenced with BigDye terminator cycle sequencing kit according to the manufacturer's protocol (Applied Biosystems, Calif.). Sequencing reactions were analyzed on an ABI 3100 automated sequencer.

\section{Construct design and zebrafish injections}

The normal TDGF 1 cDNA was generated by amplification from a human ovary 5'-STRETCH cDNA library (Clontech) and directionally subcloned into the pSP64poly(A) (Promega) expression vector. The entire coding region was sequence verified bidirectionally and confirmed to correspond to TGDF1. Each of the two missense mutations was individually introduced by site-directed mutagenesis. Verification that only the intended mutations and no other alterations were present was established by bi-directional sequencing. Plasmids used in the functional assays were linearized with EcoR1 and sense-strand RNA was synthesized by the SP6 Message machine kit (Ambion); RNA was directly injected into the embryos in the amounts indicated in Fig. 2 and the resulting phenotypes were scored by direct microscopic inspection.

\section{Nomenclature}

Gene mutation nomenclature used in this article follows the recommendations of den Dunnen and Antonarakis (2001). Gene symbols used in this article follow the recommendations of the HUGO Gene Nomenclature Committee (Povey et al. 2001)

\section{Results}

In this study, we have identified two apparently unique heterozygous missense mutations in TDGF1 by utilizing denaturing HPLC and the same panel of 83 familial and 327 sporadic DNA samples from patients with HPE previously screened for $C F C 1$ (Bamford et al. 2000). The first mutation, $\mathrm{P} 125 \mathrm{~L}$, is present in the CFC domain of TDGF 1 from a patient with developmental delay and a dysplastic forebrain (Fig. 1a-c). In addition, midline anomalies, such as absence of the septum pellucidum and a hypoplastic corpus callosum, are present in this patient. We determined that his mother was not a mutation carrier; however, the father was not available for analysis.

Persistent developmental delay led to the performance of an MRI scan at 15 months of age on this first patient. The magnetic resonance imaging study included sagittal and coronal $\mathrm{T} 1$ weighted images, in addition to axial T2 weighted images. The images (reviewed by one of us, A.J.B.) revealed a cerebrum characterized by marked ventriculomegaly and complete absence of the septum pellucidum. The interhemispheric fissure and falx cerebri were normally formed, excluding the diagnosis of holoprosencephaly. The volume of the cerebral white matter was very diminished. The cerebral cortex appeared to have normal thickness, and the gyral pattern was grossly normal, other than the sylvian fissures, which were shallow and uncovered as the frontal, temporal, and parietal opercula were very hypoplastic. The hippocampi and optic nerves were small. Images of the posterior fossa showed a small pons with a vertical midline anterior cleft, suggesting absence of transverse pontine fibers. The cerebellar hemispheres were normal, and the vermis was small, but had a grossly normal number of lobules.

The second mutation, V159M, is near the carboxy terminus of the protein, which is thought to mediate its association with the cell membrane (Minchiotti et al. 2000). This variation was found in a patient with sporadic semilobar HPE and characteristic HPE facial findings, including hypotelorism, microcephaly, and midline clefting of both the lip and palate. In this case, we could also demonstrate by sequencing that the mother was not a carrier, but the father was not available for analysis, since the family was no longer in contact with the referring physicians. Neither of these mutations is detectable in over 200 normal control chromosomes, nor have they been identified in other patient groups presently being examined (data not shown). Additional variants found in the HPE samples, as well as normal controls, include V22A, Y43D, E56E, G116G, and L152L, and are presumed to be polymorphisms.

To test the function of these unique TDGF1 mutations, we utilized the same zebrafish rescue assay as was previously performed for mutations in $C F C l$, since zebrafish lacking oep activity can be rescued by all mammalian EGF-CFC proteins (Gritsman et al. 1999; Bamford et al. 2000). We generated a control TDGF1 construct by amplification from an ovary cDNA library and introduced the identified mutations via site-directed mutagenesis. Wildtype human TDGF1 rescues oep mutants, resulting in zebrafish with two separated eyes and a hatching gland (Fig. 2e, f). In contrast, the P125L construct was completely inactive. Injected zebrafish are cyclopic, and lack the developing hatching gland (Fig. $2 \mathrm{i}, \mathrm{j}$ ), providing strong evi- 
Fig. 1a-f Mutations identified in TDGF1. a Two-year old male child with developmental delay, relatively minor craniofacial findings, small head size (tracking at the third percentile, compared with his weight at the tenth percentile) and a P125L mutation in TDGF1. MRI scans of this individual show a prominent single ventricle and subarachnoid space, absence of the septum pellucidum, with markedly decreased subcortical white matter (b) and marked thinning of the corpus callosum (c). Additional findings include hypoplastic hippocampi, small optic nerves and chiasm, and a ventral defect in the pons (data not shown). Sequence analysis with nucleotide and codon change of $\mathrm{P} 125 \mathrm{~L}$ is shown in (d) and V159 $\mathrm{M}$ in (e). f Sequence alignment of the $\mathrm{CFC}$ domain and exon 6 among EGF-CFC family members and positions of variations identified in TDGF1. Positions of variants are highlighted in red, while conserved residues are highlighted in blue. The functional CFC domain is indicated by a solid blue line
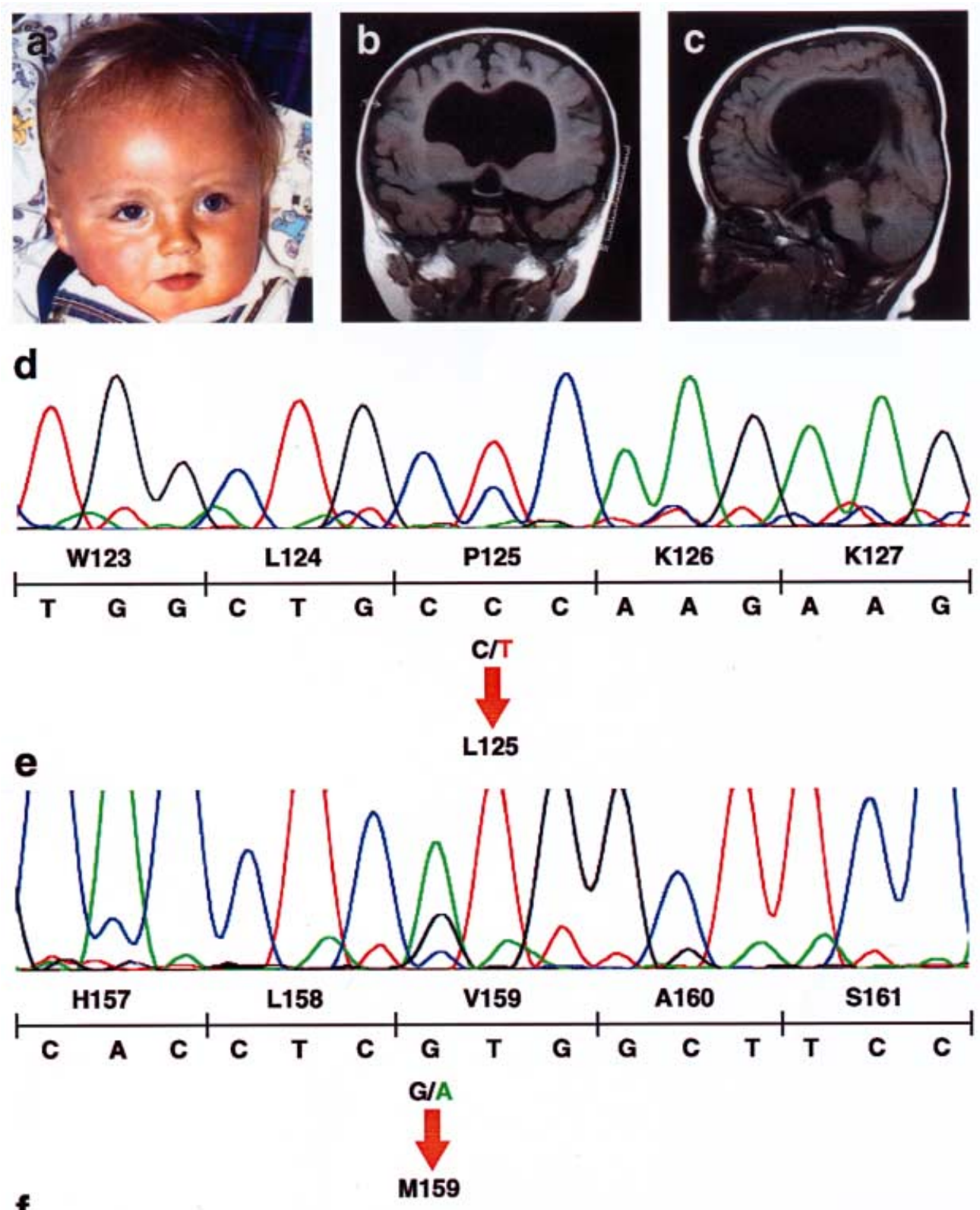

f

\begin{tabular}{|c|c|c|}
\hline \multirow[b]{2}{*}{$\begin{array}{l}\text { Human } \\
\text { Mouse } \\
\text { Human } \\
\text { Mouse } \\
\text { Fish }\end{array}$} & \multirow[b]{2}{*}{$\begin{array}{l}\text { TDGF-1 } \\
\text { Tdgi-1 } \\
\text { CFC1 } \\
\text { Cfc1 } \\
\text { oep }\end{array}$} & \multirow[b]{2}{*}{$\begin{array}{l}\text { CGSVPHDTWLPKKCSLCKCWHGQLRCFPQAFLPG } \\
\text { CGS I LHGTWLPKKCSLCRCWHGQLHCLPQTFLPG } \\
\text { CGALEHGAWT LRACHLCRCIFGALHCLPLQTPDR } \\
\text { CGALGHGAWTLHSCRLCRCIFSALYCLPHQTFSH } \\
\text { CGVIPHGEWVOKGCSYCRCGYGLLHCFPHVFSKD }\end{array}$} \\
\hline & & \\
\hline $\begin{array}{l}\text { Human } \\
\text { Mouse } \\
\text { Human } \\
\text { Mouse } \\
\text { Fish }\end{array}$ & $\begin{array}{l}\text { TDGF-1 } \\
\text { Tdgf-1 } \\
\text { CFC1 } \\
\text { Cfc1 } \\
\text { oep }\end{array}$ & $\begin{array}{l}\overline{C D G L V M D E H L V A S R T P E L P P S A R--T T T F M L V G I ~} \\
\text { CDGHVMDQDLKASRTPCQTPSV-- TTTFMLAGA } \\
\text { CDPKDFLASHAHGPSAGGAPSLLLLLPCALLHRL } \\
\text { CDLKSFLSSGARGSRECSIPSLLLLVLCLLLQ-- } \\
\text { CDDSOEVRWHRSGSLRTLSSTIVMFATFILH--- }\end{array}$ \\
\hline $\begin{array}{l}\text { Human } \\
\text { house } \\
\text { Human } \\
\text { house }\end{array}$ & $\begin{array}{l}\text { TDGF-1 } \\
\text { Tdgf-1 } \\
\text { CFC1 } \\
\text { Cfc1 }\end{array}$ & 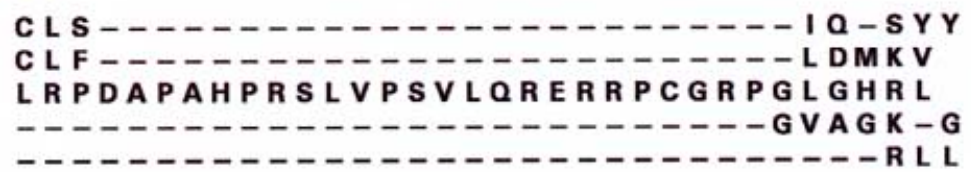 \\
\hline
\end{tabular}



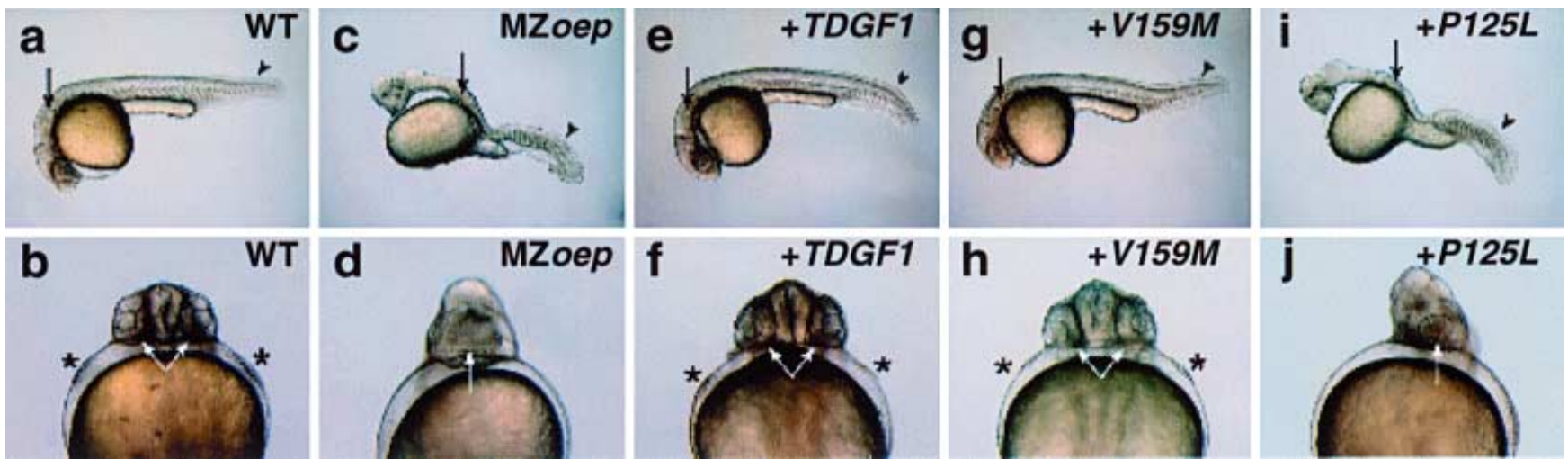

Fig. 2a-j Activities of TDGF1 mutations in zebrafish embryos. Embryos shown are at 30-36 h post fertilization. a, b Wild-type embryo. a Lateral view. Note the distance between the ear (arrow) and the tail somites (arrowhead). b Ventral view. Note the separated eyes (arrows) and the presence of the hatching gland (*). c, d Uninjected MZoep embryo. c Lateral view. The lack of trunk results in a short distance between the ear (arrow) and the few tail somites that form (arrowhead). d Ventral view. Only a cyclopic eye (arrow) forms and the hatching gland is absent. e, f MZoep embryo injected with TDGF1. e Lateral view of a rescued embryo. Head and trunk mesoderm and endoderm are rescued by the injected RNA, and the proper distance between the ear (arrow) and the tail somites (arrowhead) is restored. $\mathbf{f}$ Ventral view of a rescued embryo. Note the rescue of the eyes (arrows) and presence of hatching gland (*) similar to wild-type. $\mathbf{g}$, $\mathbf{h}$ Lateral and ventral views of an MZoep embryo injected with V159M showing complete rescue. $\mathbf{i}, \mathbf{j}$ Lateral and ventral views of an MZoep embryo injected with P125L showing a lack of rescue. Arrows, arrowheads, and symbols in $(\mathbf{g}, \mathbf{h}, \mathbf{i}, \mathbf{j})$ are as those in $(\mathbf{a}, \mathbf{b}, \mathbf{c}, \mathbf{d})$

dence that the CFC domain is required for human TDGF1 function. Based on the Garnier-Robson-Osguthorpe Secondary Structure Prediction algorithm, the P125L variant is predicted to alter the secondary structure of TDGF1 from a turn to an alpha helix between residues 121 and 125. In contrast, injection of a V159M construct results in the rescue of the oep phenotype to an extent that is similar to wild-type TDGF1 (Fig. 2g, h; see also Table 2). A similar analysis of the predicted secondary structure for the V159M alteration indicates that a four-amino-acid helical region is extended by two amino acids by this variation. The significance of this change is unclear. Therefore, the $\mathrm{V} 159 \mathrm{M}$ variation is likely to be an uncommon conservative missense change.

\section{Discussion}

Here we show for the first time that a loss-of-function mutation in the CFC domain disrupts TDGF1 activity in humans. We conclude that the failure of the mutated form of TDGF1 containing the $\mathrm{P} 125 \mathrm{~L}$ variation to rescue the oep phenotype is attributable to either instability of the mRNA or, more likely, a change in the structure of the CFC domain such that it can no longer interact with receptor components. Further biochemical and genetic studies would be needed to determine whether or not the $\mathrm{P} 125 \mathrm{~L}$ variation has any additional potential dominant effects. It is also interesting to note that while this $\mathrm{P} 125$ residue is conserved between human and mouse TDGF1, it is not conserved across family members or species. In fact, an L residue is present in the corresponding position in human and mouse CFC1. It will take detailed structural studies to clarify the folding requirements of the CFC domain among family members. Clearly, mutations in conserved regions of the EGF-CFC family, such as the EGF-like and CFC
Table 2 Zebrafish rescue assay injection results of TDGFI constructs

\begin{tabular}{|c|c|c|c|c|c|}
\hline mRNA injection & Amount $^{\mathrm{a}}$ & $n$ & $\begin{array}{l}\text { Complete } \\
\text { rescue }^{b}\end{array}$ & $\begin{array}{l}\text { Partial } \\
\text { rescue }\end{array}$ & No rescue ${ }^{d}$ \\
\hline Uninjected control & $0 \mathrm{pg}$ & 44 & $0(0 \%)$ & $0(0 \%)$ & $44(100 \%)$ \\
\hline oep & $50 \mathrm{pg}$ & 92 & $82(89 \%)$ & $10(11 \%)$ & $0(0 \%)$ \\
\hline$T D G F 1$ & $50 \mathrm{pg}$ & 28 & $6(21 \%)$ & $22(79 \%)$ & $0(0 \%)$ \\
\hline$V 159 M$ & $50 \mathrm{pg}$ & 81 & $9(11 \%)$ & $58(72 \%)$ & $14(17 \%)$ \\
\hline$P 125 L$ & $50 \mathrm{pg}$ & 40 & $0(0 \%)$ & $0(0 \%)$ & $40(100 \%)$ \\
\hline Uninjected control & $0 \mathrm{pg}$ & 32 & $0(0 \%)$ & $0(0 \%)$ & $32(100 \%)$ \\
\hline oеp & $100 \mathrm{pg}$ & 63 & $61(97 \%)$ & $2(3 \%)$ & $0(0 \%)$ \\
\hline$T D G F 1$ & $100 \mathrm{pg}$ & 41 & $6(15 \%)$ & $35(85 \%)$ & $0(0 \%)$ \\
\hline V159M & $100 \mathrm{pg}$ & 65 & $1(1 \%)$ & $60(93 \%)$ & $4(6 \%)$ \\
\hline$P 125 L$ & $100 \mathrm{pg}$ & 24 & $0(0 \%)$ & $0(0 \%)$ & $24(100 \%)$ \\
\hline оер & $10 \mathrm{pg}$ & 15 & $15(100 \%)$ & $0(0 \%)$ & $0(0 \%)$ \\
\hline$T D G F 1$ & $10 \mathrm{pg}$ & 11 & $0(0 \%)$ & $1(9 \%)$ & $10(91 \%)$ \\
\hline$V 159 M$ & $10 \mathrm{pg}$ & 37 & $0(0 \%)$ & $1(3 \%)$ & $36(97 \%)$ \\
\hline
\end{tabular}


domains, as well as the carboxy terminus, affect normal protein function, emphasizing that these domains are essential for the activity of human protein members of this family. These conclusions are also supported by recent biochemical studies defining the specific roles of EGF and CFC domains (Yeo and Whitman 2001).

The patient with the P125L mutation has severe midline brain anomalies compatible, but not prototypical of HPE. Specifically, the HPE-like features include the absence of the septum pellucidum, hypoplasia of the corpus callosum and optic nerves and chiasm, which are noted from the MRI performed at 15 months of age. All of these findings can be seen as microforms of HPE. Nevertheless, the presence of an intact interhemispheric fissure precludes the formal diagnosis of HPE. In addition, the marked ventriculomegaly, small hippocampi and anterior cleft of the pons are not typical of HPE. It is unclear if the reduced deep white matter and dysplasia of the sylvian fissures are primary findings or secondary to hydrocephalous, which was first noted by ultrasound at 18 weeks of gestation. Serial scans through the remainder of the pregnancy were interpreted as consistent with lobar HPE. It is now apparent that this mis-classification as HPE could be attributed to the relative lack of precision and anatomic detail of the ultrasound procedure.

It is likely that some of the clear differences between the phenotype of this patient and those more typical of HPE reflects a difference in the mechanism of disease between factors that directly act on the forebrain, such as Sonic Hedgehog, compared with those acting more indirectly on axial midline development, such as Nodal. Further mutations would need to be identified and analyzed before a clear genotype-phenotype correlation can be made.

In summary, our results suggest that mutations in the CFC domain of TDGF1 can affect human forebrain development. We have previously shown that mutations in another human EGF-CFC gene, $C F C 1$, are associated with laterality defects (Bamford et al. 2000). Previous studies in zebrafish have shown that the EGF-CFC gene one-eyed pinhead is required for the development of both the forebrain and correct laterality (Schier et al. 1997; Gritsman et al. 1999; Yan et al. 1999). Taken together, these results indicate that the dual role for oep in both processes in fish is divided between the related genes TDGF1 and CFC1 in humans. Finally, it is interesting to note that only one mutation with altered function was identified among over 400 patients with HPE in either $C F C 1$ or TDGF1. Given the central importance of Nodal signaling in the pathogenesis of cyclopia inferred from animal model systems, we were surprised to find that similar mutations in humans are so rare. A likely explanation is that if and when loss of function mutations occurs in these genes during fetal development the probability of spontaneous pregnancy loss is substantially increased. Since our sample of patients only represents live-born infants, there is an inherent bias in the genes identified that will be associated with HPE.
Acknowledgements We sincerely thank J.D. Karkera for assistance with protein prediction algorithms, W.B. Dobyns for reading the brain scans of the patients. R.D.B. is supported by a Cancer Research Fund Fellowship of the Damon Runyon-Walter Winchell Foundation. A.F.S. is a Scholar of the McKnight Endowment Fund for Neuroscience and the Irma T. Hirschl Trust. This work is supported by grants from the NIH (A.F.S.) and by the Division of Intramural Research, NHGRI, NIH (M.M.).

\section{References}

Bamford RN, Roessler E, Burdine RD, Saplakoglu U, dela Cruz J, Splitt M, Towbin J, et al (2000) Loss-of-function mutations in the EGF-CFC gene $\mathrm{CFCl}$ are associated with human left-right laterality defects. Nat Genet 26:365-369

Burdine RD, Schier AF (2000) Conserved and divergent mechanisms in left-right axis formation. Genes Dev 14:763-776

Conlon FL, Lyons KM, Takaesu N, Barth KS, Kispert A, Herrmann B, Robertson EJ (1994) A primary requirement for nodal in the formation and maintenance of the primitive streak in the mouse. Development 120:1919-1928

den Dunnen JT, Antonarakis SE (2001) Nomenclature for the description of human sequence variations. Hum Genet 109:121124

Ding J, Yang L, Yan Y-T, Chen A, Desai N, Wynshaw-Boris A, Shen MM (1998) Cripto is required for correct orientation of the anterior-posterior axis in the mouse embryo. Nature 395: $702-707$

Feldman B, Gates MA, Egan ES, Dougan ST, Rennebeck G, Sirotkin HI, Schier AF, et al (1998) Zebrafish organizer development and germ-layer formation require nodal-related signals. Nature 395:181-185

Gritsman K, Zhang J, Cheng S, Heckscher E, Talbot WS, Schier AF (1999) The EGF-CFC Protein One-Eyed Pinhead Is Essential for Nodal Signaling. Cell 97:121-132

Gritsman K, Talbot WS, Schier AF (2000) Nodal signaling patterns the organizer. Development 127:921-932

Minchiotti G, Parisi S, Liguori G, Signore M, Lania G, Adamson ED, Lago CT, et al. (2000) Membrane Anchorage of Cripto protein by glycosylphosphatidlyinositiol and its distribution during early mouse development. Mech Dev 90:133-142

Muenke M, Beachy P (2001) Holoprosencephaly. In: Scriver CR, Beaudet AL, Sly WS, Valle D, Childs B, Kinzler KW, Vogelstein B (eds) The metabolic and molecular bases of inherited disease. 250: Holoprosencephaly. McGraw-Hill, pp 6203-6230

Nanni L, Ming JE, Bocian M, Steinhaus K, Bianchi DW, DieSmulders C, Giannotti A, Imaizumi K, Jones KL, Campo MD, Martin RA, Meinecke P, Pierpont ME, Robin NH, Young ID, Roessler E, Muenke M (1999) The mutational spectrum of the Sonic Hedgehog gene in holoprosencephaly: SHH mutations cause a significant proportion of autosomal dominant holoprosencephaly. Hum Mol Genet 8:2479-88

Povey S, Lovering R, Bruford E, Wright M, Lush M, Wain $\mathrm{H}$ (2001) The HUGO Gene Nomenclature Committee (HGNC). Hum Genet DOI 10.1007/s00439-001-0615-0

Roessler E, Muenke M (2001) Midline and laterality defects: left and right meet in the middle. Bioessays 23:888-900

Schier AF, Shen MM (2000) Nodal signalling in vertebrate development. Nature 403:385-389

Schier AF, Talbot WS (2001) Nodal signaling and the zebrafish organizer. Int J Dev Biol 45:289-297

Schier AF, Neuhauss SCF, Helde KA, Talbot WS, Driever W (1997) The one-eyed pinhead gene functions in mesoderm and endoderm formation in zebrafish and interacts with no tail. Development 124:327-342

Shen MM, Schier AF (2000) The EGF-CFC gene family in vertebrate development. Trends Genet 16: 303-309 
Shen MM, Wang H, Leder P (1997) A differential display strategy identifies Cryptic, a novel EGF-related gene expressed in the axial and lateral mesoderm during mouse gastrulation. Development 124:429-442

Strahle U, Jesuthasan S, Blader P, Garcia-Villalba P, Hatta K, Ingham PW (1997) one-eyed pinhead is required for development of the ventral midline of the zebrafish (Danio rerio) neural tube. Genes Funct 1:131-148

Yan, Y-T, Gritsman K, Ding J, Burdine RD, Corrales JD, Price SM, Talbot WS, et al (1999) Conserved requirement for $E G F-C F C$ genes in vertebrate left-right axis formation. Genes Dev 13: $2527-2537$
Yeo C-Y, Whitman M (2001) Nodal signals to Smads through Cripto-Dependent and Cripto-independent mechanisms. Mol Cell 7:949-957

Zhang J, Talbot WS, Schier AF (1998) Positional cloning identifies zebrafish one-eyed pinhead as a permissive EGF-related ligand required during gastrulation. Cell 92:241-251

Zhou X, Sasaki H, Lowe L, Hogan BL, Kuehn MR (1993) Nodal is a novel TGF-beta-like gene expressed in the mouse node during gastrulation. Nature 361:543-547 